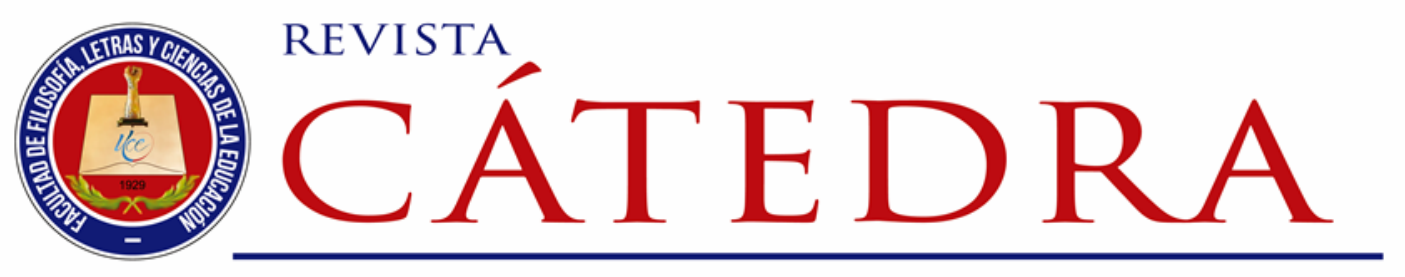

\title{
Construcción de la sexualidad infantil en el ambiente familiar
}

\section{Construction of infant sexuality in the family environment \\ Martha Quirola-Larrea \\ Universidad Central del Ecuador, Quito, Ecuador \\ marthaquirolal@hotmail.com \\ https://orcid.org/0000-0003-1637-6507}

Cecilia Jaramillo-Jaramillo

Universidad Central del Ecuador, Quito, Ecuador

cjaramilloj@uce.edu.ec

https://orcid.org/0000-0003-0182-9315

(Recibido: 09/03/2020; Aceptado: 15/03/2020; Versión final recibida: 23/04/2020)

Cita del artículo: Quirola-Larrea, M. y Jaramillo-Jaramillo, C. (2020). Construcción de la sexualidad infantil en el ambiente familiar. Revista Cátedra, 3(2), 52-72.

\section{Resumen}

La construcción de la sexualidad infantil se halla invisibilizada en la sociedad. Las personas adultas consideran que los niños y niñas infantes son seres asexuados y subsisten los tabúes alrededor de la sexualidad humana. Ocultar el tema mantendrá la ignorancia sobre la sexualidad infantil. La investigación sobre la sexualidad generalmente ha estado dirigida hacia la adolescencia como supuesta etapa en la que la sexualidad aparece en el desarrollo humano. El objetivo es verificar si mediante un proceso formativo cambian las formas en cómo los padres/madres de familia procuran formación e información sobre la sexualidad a sus hijos e hijas. Se implementó un programa de sexualidad infantil con representantes legales de un Centro de Desarrollo Integral para la Primera Infancia de la ciudad de Quito. Esta investigación es de tipo cuasi experimental. Se aplicaron encuestas de entrada y salida a los actores directos e indirectos del programa, divididos en grupos experimental y de control. La conclusión final es que el programa tuvo un impacto significativo en el cambio de perspectiva de los padres/madres de familia. Incluye las prácticas de diversas posibilidades de educación sexual con sus hijos e hijas. De igual forma, se presenta la posibilidad de educar a progenitores en los ámbitos escolar y comunitario para mejorar la calidad de vida y de gestión familiar.

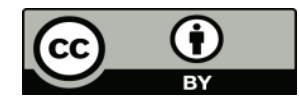


Palabras clave

Creencias, familia, género, infancia, sexualidad.

\section{Abstract}

The construction of infant sexuality is invisible in society. Adults consider infant children (boys and girls) to be asexual and taboos about human sexuality persist. Hiding the topic will maintain ignorance about child sexuality. Research on sexuality has focused on puberty and adolescents as periods of sexual development in humans. The objective is to verify if, through a training process, the ways in which parents provide training and information about sexuality to their sons and daughters change. An infant sexuality program was implemented for the legal guardians of the children in an early childhood program in the city of Quito. This research is quasi-experimental. Survey will be given at the beginning and end of the program to people directly and indirectly responsible of the program. Additionally, there will be an experimental and a control group. The final conclusion is that the program had a significant impact on the change of perspective of the parents. It includes the practices of various possibilities of sexual education with their sons and daughters. In the future, there is the possibility to educate parents in the school and community environment to improve the quality of life and family management.

Key words

Beliefs, family, gender, infancy, sexuality.

\section{Introducción}

La literatura científica sobre la sexualidad infantil es limitada. El énfasis colocado en la adolescencia como principal foco de atención investigativa de la sexualidad humana, ha restringido la indagación del período de la niñez. Un factor de incidencia esencial es que existe la idea de que los niños y niñas son seres asexuados (Hernández y Jaramillo, 2003). Así, la educación sexual infantil, como eje de gestión de algunos proyectos y programas, se desenvuelve con preponderancia en la institucionalidad educativa. Cuando se implementa en el ambiente familiar generalmente es de modo complementario y subsidiario al escolar.

Las competencias familiares se aplican de una manera incipiente o sencillamente no se ejecutan en lo que se refiere a la formación e información sobre la sexualidad. Por ello, la recurrencia de padres y madres de familia de ocultar los órganos genitales y su fisiología en un lenguaje coloquial, inexacto e inapropiado. Las prácticas de enseñanza que los miembros adultos familiares aplican en la cotidianidad contribuyen a una formación llena de temores, sentimiento de vergüenza, culpabilidad y creencias infundadas que más bien perturban e inhiben las expresiones sexuales.

El propósito investigativo incluye caracterizar el empleo del lenguaje y las estrategias educativas materno-parentales en la educación sexual infantil. También es necesario ver su influencia en los conocimientos, creencias y actitudes de la sexualidad de infantes de 4 a 5 años de edad.

Esta investigación abarcó un programa de sexualidad infantil desarrollado con las madres y padres de familia de un Centro de Desarrollo Integral para la Primera Infancia de sostenimiento fiscal. El planteamiento hipotético fue que la aplicación de este programa influiría en las estrategias parentales aplicadas en el ambiente familiar para incidir en las nociones que las niñas y niños puedan aprenderlo o asumirlo.

Licencia Creative Commons Atribución 4.0 Internacional (CC BY 4.0)

Revista Cátedra, 3(2), pp. 52-72, mayo-agosto 2020. e-ISSN: 2631-2875

https://doi.org/10.29166/10.29166/catedra.v3i2.2184 
El documento se presentó como trabajo investigativo en la Maestría de Educación Inicial en la Facultad de Filosofía, Letras y Ciencias de la Educación de la Universidad Central del Ecuador. Contiene algunos elementos teóricos para encuadre conceptual y el proceso metodológico aplicado. Luego se exponen los resultados obtenidos y su respectivo análisis y discusión. Finalmente, se tiene las conclusiones y la bibliografía consultada.

\section{Conceptos relacionados}

\subsection{Referentes históricos y legales en la institucionalidad educativa ecuatoriana}

En el Ecuador, las políticas y programas que se han ejecutado en cuanto a la educación sexual tienen su mirada en el estudiantado adolescente. El esfuerzo más importante en materia de educación en la sexualidad realizado por el estado ecuatoriano, fue a través del Ministerio de Educación. Se desarrolló a partir de la Ley sobre la Educación de la Sexualidad y el Amor promulgada en el Registro Oficial 285, en 1998, según el Acuerdo Ministerial 403 (Registro Oficial 386, pág. 1). Su organización se efectuó con el Plan Nacional de Educación para la Sexualidad y el Amor, conforme a dicha ley, en al año 2000. La ejecución se formalizó a través del Programa Nacional de Educación de la Sexualidad y el Amor (PRONESA), en todas las Direcciones Provinciales de Educación. De este modo, se procuró su implementación en las instituciones escolares de los niveles básico y bachillerato del sistema educativo.

Posterior a esta política nacional, se han desarrollado otros procesos que se han plasmado en normativas sustantivas que describen esfuerzos en cuanto a su cumplimiento. Así se tiene: Acuerdo Ministerial No. 3393: Reglamento especial de procedimientos y mecanismos para el conocimiento y tratamiento de los delitos sexuales en el sistema educativo (Registro Oficial 431, 2004, pág. 4-8). Acuerdo Ministerial No. 403: Institucionalizar la educación sexual en los establecimientos educativos fiscales, particulares, fiscomisionales y municipales del país a través del PRONESA (Registro Oficial 386, 2006, pág. 14-15). Decreto Ejecutivo No. 620: Declaración como Política de Estado del "Plan Nacional para la erradicación de la violencia de género hacia la niñez, adolescencia y mujeres" (Registro Oficial 171, 2007, pág. 3-4). Acuerdo Ministerial 062: Plan nacional para la erradicación de los delitos sexuales en el ámbito educativo (Ministerio de Educación y Cultura, 2006).

El Acuerdo Interministerial No. 0247-2018 del año 2018: Política intersectorial de prevención del embarazo en niñas y adolescentes ecuador 2018 - 2025 (Registro Oficial 529,2018 , pág. 1-44). En su extracto se señala que el embarazo en niñas y adolescentes constituye un problema social y de salud pública. Se evidencia la desigualdad, la injusticia social y una clara violación de los derechos humanos, con un impacto social, económico y cultural invalorable. Participan de esta política los Ministerios de Salud, Educación, Justicia e Inclusión Económica y Social.

El Programa Educando en Familia impulsado por el Ministerio de Educación 2015 aborda con un enfoque de integralidad la educación de la sexualidad y afectividad. Es el esfuerzo más amplio e inclusivo que tiene la instancia ministerial para el tema y se halla en plena ejecución.

Una evaluación preliminar de las acciones, procesos e implementación de las normativas legales señaladas, indica que hay una ejecución parcial de las políticas, que se debe a la escasez de recursos financieros necesarios para su aplicación, baja disponibilidad del talento humano requerido y la insuficiente cobertura de dichos programas. Estos elementos hacen que la problemática social subyacente, se halle contenida en algunas instituciones y sectores educativos y, en otros, se haya incrementado. Ninguna política se ha dirigido hacia la preparación de padres y madres de familia y estudiantes de educación inicial. Las Licencia Creative Commons Atribución 4.0 Internacional (CC BY 4.0) 
decisiones estatales para atender la formación de progenitores y del alumnado marcan una ausencia de partida en el proceso formativo de estos sujetos sociales. Un proceso integral en todos los subniveles educativos y con el involucramiento de los actores fundamentales, se podrá lograr en una educación sexual de calidad.

\subsection{Estado de la cuestión}

Para este estudio, el ambiente familiar es el espacio en el que se desenvuelven las enseñanzas sobre nociones de sexualidad aplicadas por madres y padres. Las formas y estrategias se basan en sus propios saberes que incluye los conocimientos, creencias y actitudes alrededor del tema. El entorno familiar esta permeado por elementos de la cultura comunitaria y social que inciden en su accionar educativo. La investigación tiene entre sus principios el enfoque de género como una categoría conceptual y analítica. Permite comprender, explicar y transformar las relaciones de inequidad, dominación, discriminación y violencia que existen entre los hombres y las mujeres. Esto no sólo a partir del sexo sino en interrelación con la edad, la etnia, la clase, la opción sexual según guía para la inclusión del MINEDUC-VVOB (2019). El género se asume como el "conjunto de prácticas, creencias, representaciones y prescripciones sociales que surgen entre los integrantes de un grupo humano en función de una simbolización de la diferencia anatómica entre hombres y mujeres." (Lamas, 2000, pág. 2). Este concepto da cuenta de la forma cómo se socializan los seres humanos en función del sexo con el que nacen.

Según Suárez, los estereotipos son preconcepciones, ideas simplificadas y generalizadas que se tiene sobre un grupo de personas. Conforme a la guía del MINEDUC-VVOB (2019) los estereotipos de género son creencias caricaturales respecto de los grupos femeninos y masculino (Suárez 2016). El androcentrismo es una visión del mundo referenciado alrededor del varón en términos masculinos y reconstrucción de lo sociocultural desde esa perspectiva. Contribuyen a la desvalorización de los primeros y a la valoración o sobrevaloración de los segundos, de acuerdo con orden social desigual entre los sexos.

Es importante precisar que el sexo tiene que ver con las características biológicas, anatómicas, morfológicas, cromosómicas y fisiológicas que definen a hombres y mujeres. La orientación sexual determina el sexo de las personas por las que se siente atracción, deseo, amor e interés (MINEDUC-VVOB, 2019). En este campo hay que considerar a la homofobia que es el temor u odio hacia los hombres y mujeres homosexuales. La identidad sexual "incluye la manera cómo la persona se identifica como mujer o como hombre, o como una combinación de ambos, y la orientación sexual de la persona" (OMS/OPS, 2000, citado por Alcántara, 2012, pág. 1). La identidad de género "tiene que ver con el sentirse masculino o femenino de las personas y sus experiencias propias y profundas." (Consejo Nacional para la Igualdad de Género, 2018, pág. 34). La idea de partida entonces es que el sexo equivale a lo biológico y el género corresponde a lo cultural.

La heteronormatividad se define como "el régimen político, social, filosófico y económico generador de violencias hacia todas aquellas personas que no seguimos un patrón de género, de sexualidad, de prácticas y deseos asociados a la heterosexualidad" (Moreno, 2019, pág. 6). Las relaciones inequitativas desde el género se encuentran normalizadas y aceptadas en la sociedad. Entre los conceptos que se asumen está el de androcentrismo que se refiere "a la visión del mundo que sitúa al hombre como centro de todas las cosas, construye una mirada masculina como universal y única posible, y conlleva la invisibilidad de las mujeres y de su mundo." (Morales, 2013, pág. 3). Desde esta posición los varones son sujetos históricos y sociales de acción, entre tanto las mujeres son más objetos que sujetos, pues se actúa sobre ellas. Antropológicamente, el patriarcado se entiende como "una organización del mundo en la cual los hombres y lo masculino se erigen como dominantes

Licencia Creative Commons Atribución 4.0 Internacional (CC BY 4.0)

Revista Cátedra, 3(2), pp. 52-72, mayo-agosto 2020. e-ISSN: 2631-2875

https://doi.org/10.29166/10.29166/catedra.v3i2.2184 
y se constituye en sistema de creencias e ideas que estructuran la forma en que vemos y entendemos el mundo." (Rosso, 2016, pág. 2). La imposición de lo heterosexual sobre las otras identidades sexuales se ha naturalizado en la sociedad. De igual manera los patrones culturales que imponen el poder del hombre sobre la mujer en general.

Finalmente, se toma el concepto de sexualidad de la Organización Mundial de la Salud (2018) que la define como

Un aspecto central del ser humano que está presente a lo largo de su vida. Abarca el sexo, las identidades y los roles de género, la orientación sexual, el erotismo, el placer, la intimidad y la reproducción. Se siente y se expresa a través de pensamientos, fantasías, deseos, creencias, actitudes, valores, comportamientos, prácticas, roles y relaciones. Si bien la sexualidad puede incluir todas estas dimensiones, no todas ellas se experimentan o expresan siempre. La sexualidad está influida por la interacción de factores biológicos, psicológicos, sociales, económicos, políticos, culturales, éticos, legales, históricos, religiosos y espirituales (pág. 3).

Los matices que cada uno de estos conceptos y de otros que se ligan, son entendidos desde la inclusividad y el enfoque de derechos. Así, el marco de conceptos ayuda a entender una realidad socioeducativa específica como es la construcción de la sexualidad infantil en el ámbito familiar. Este espacio social incluye fundamentalmente a los padres/madres como referentes afectivos y de saberes que tienen niños y niñas. Se asume como "construcción» pues diversos aspectos de la sexualidad son aprendidos.

\section{Metodología}

\subsection{Población y muestra}

La población estudiantil del Centro para el Desarrollo Integral para la Primera Infancia Virginia Larenas está constituida por 438 niños y niñas. La de padres/madres de familia y representantes legales es de 430 personas. La muestra estuvo formada por 20 representantes legales del grupo experimental y 20 del grupo control, que equivalen al $9.3 \%$ de la población. El estudiantado del grupo experimental estuvo compuesto por 20 niños y niñas de 4-5 años de edad, correspondiente al grupo experimental de progenitores. El grupo control fueron 20 estudiantes de distintos paralelos tomados aleatoriamente. Estos grupos corresponden al 9.1\% de la población. Todos los individuos de la encuesta estudiantil se hallaban en la edad de 4-5 años.

\subsection{Diseño de la investigación}

El diseño operativo de la investigación fue cuasi experimental, con la intervención de un grupo experimental y un grupo control. La conformación de estos grupos se hizo en base a la nómina de participantes entregada por la institución educativa. Se determinó el desarrollo del programa de educación sexual infantil con el grupo experimental. También la aplicación de las encuestas de entrada y salida (testy retest) a los dos grupos. El test y retest son cuestionarios que investigan sobre los conocimientos, creencias y actitudes sobre la sexualidad infantil. Estos instrumentos fueron utilizados para determinar la incidencia del programa formativo aplicado.

La aplicación del test y retest del grupo experimental fue con las mismas personas que participaron en el programa. La aplicación del retest del grupo control fue con personas distintas a las que contestaron el test, aunque de la misma institución educativa. Así, la caracterización es que se trata de un diseño de pretest - postest con grupo de control no Licencia Creative Commons Atribución 4.0 Internacional (CC BY 4.0) 
equivalente (Murillo, s.f. pág. 25). Los instrumentos para la recolección de datos fueron los cuestionarios. En el caso de los progenitores está compuesta de 36 ítems de diferente tipo: lista de cotejo, escala valorativa tipo Likert y preguntas abiertas. Para el estudiantado, tuvo 18 ítems con preguntas tipo lista de cotejo y abiertas. Se realizaron registros anecdóticos de aspectos relevantes del programa de educación en sexualidad infantil aplicado al grupo experimental de madres y padres de familia.

\subsection{Programa de intervención: Programa de Educación Sexual en Familia-PSIF}

El Programa de Educación Sexual en Familia (PSIF) es un proceso de educación sexual para padres/madres de familia de niños y niñas infantes. Fue diseñado por las autoras del presente artículo. Contiene la justificación, objetivos, lineamientos pedagógicos y metodológicos generales, ciclo de talleres y las referencias bibliográficas. Hay un ambiente social de sobreexposición mediática sobre la sexualidad por lo que se requiere la formación sexual en los espacios naturales a la niñez. El principio pedagógico asumido es que el entorno familiar es el ideal para alcanzar una educación integral de la niñez. El programa facilita información y guía sobre aspectos que los progenitores no saben cómo transmitir y toma en cuenta que algunos conocimientos, creencias y actitudes que son producto del desconocimiento respecto al desarrollo del infante.

El PSIF tiene dos objetivos: 1) Reflexionar sobre la importancia de la educación sexual en el ámbito familiar; y, 2) Identificar actitudes y acciones que los progenitores deben considerar o aplicar para que ayuden a sus hijos e hijas a conocer y respetar su cuerpo, establecer las diferencias sexuales entre hombres y mujeres, y reflexionar las diferencias entre los géneros. El ciclo formativo implica ocho talleres de 1,5 hora cada uno, lo que en conjunto da un total de 12 horas de gestión. Cada taller incluye su respectiva agenda con su título, datos específicos, objetivo, actividades, recursos, evaluación y la referencia bibliográfica específica.

\section{Resultados de la investigación}

En una primera parte, las encuestas incluyeron algunas preguntas de carácter sociodemográfico para establecer las características del grupo de representantes legales que aplicaron los instrumentos. Para este artículo se tienen los resultados correspondientes al sexo, formación académica y religión.

\begin{tabular}{lcccc}
\hline \multirow{2}{*}{ Opciones } & GE-PMF & \multicolumn{3}{c}{ GC-PMF } \\
\cline { 2 - 5 } & $\mathrm{F}$ & $\%$ & $\mathrm{f}$ & $\%$ \\
Hombre & 3 & 15 & 0 & 0 \\
Mujer & 17 & 85 & 20 & 100 \\
\hline
\end{tabular}

Cuadro 1: Sexo de padres y madres de familia participantes

En la encuesta de salida, se tiene para el Grupo Control de Padres/Madres de familia (GCPMF) el $100 \%$ de mujeres. En el Grupo Experimental de Padres/Madres de familia (GEPMF) $85 \%$ de mujeres y $15 \%$ de hombres. Existe pues una presencia mayoritaria de mujeres que es muy común en las instituciones educativas del sector (Cuadro 1).

El 70\% de participantes del GE-PMF, tiene estudios de bachillerato y el $25 \%$ universitario. El GC-PMF, afirma tener formación básica el $55 \%$, secundaria $30 \%$ y universitario $10 \%$ (Cuadro 2).

Licencia Creative Commons Atribución 4.0 Internacional (CC BY 4.0)

Revista Cátedra, 3(2), pp. 52-72, mayo-agosto 2020. e-ISSN: 2631-2875

https://doi.org/10.29166/10.29166/catedra.v3i2.2184 
Un elemento a conocer fue la relación entre las creencias y los conocimientos que tienen los padres/ madre de familia sobre la sexualidad y sus efectos en las nociones de sus hijos/hijas; de allí la importancia de saber si profesan alguna religión. El 58 \% afirman ser no creyentes y el $42 \%$ sí serlo (Cuadro 3). Las respuestas sorprenden viniendo de una sociedad como la ecuatoriana y la del sector poblacional encuestado.

\begin{tabular}{llllll}
\hline Opciones & \multicolumn{2}{l}{ GE-PMF } & \multicolumn{2}{l}{ GC-PMF } \\
\cline { 2 - 6 } & $\mathrm{f}$ & $\%$ & $\mathrm{f}$ & $\%$ \\
Ninguno & 0 & 0 & 1 & 5 \\
Básico (completo o incompleto) & 1 & 5 & 11 & 55 \\
Bachillerato (completo o incompleto) & 14 & 70 & 6 & 30 \\
Universitario (completo o incompleto) & 5 & 25 & 2 & 10 \\
\hline
\end{tabular}

Cuadro 2: Nivel de estudios de padres y madres de familia participantes

\begin{tabular}{lllll}
\hline Opciones & \multicolumn{2}{l}{ GE-PMF } & \multicolumn{2}{l}{ GC-PMF } \\
\cline { 2 - 5 } & $\mathrm{f}$ & $\%$ & $\mathrm{~F}$ & $\%$ \\
Sí & 7 & 35 & 8 & 40 \\
No & 13 & 65 & 12 & 60 \\
\hline
\end{tabular}

Cuadro 3: ¿Profesa alguna religión?

\begin{tabular}{|c|c|c|c|c|c|}
\hline \multirow{3}{*}{$\begin{array}{l}\text { Desarrollo de la } \\
\text { sexualidad } \\
\text { Edad de mediación }\end{array}$} & \multirow{2}{*}{$\begin{array}{c}\text { Ítems } \\
10\end{array}$} & \multicolumn{4}{|c|}{$\begin{array}{l}\text { Nivel de significación Padres y Madres de Familia } \\
\text { A. Grupo experimento vs Grupo control } \\
\text { B. Impacto en grupo experimento (test vs retest) }\end{array}$} \\
\hline & & $\begin{array}{c}\text { Muy } \\
\text { significativo } \\
\checkmark\end{array}$ & $\begin{array}{c}\text { Mediano } \\
\text { significativo }\end{array}$ & $\begin{array}{c}\text { Poco } \\
\text { significativo }\end{array}$ & $\begin{array}{c}\text { Nada } \\
\text { significativo }\end{array}$ \\
\hline & 11 & $\checkmark$ & & & \\
\hline $\begin{array}{l}\text { Orientación sexual } \\
\text { definida }\end{array}$ & 12 & & $\checkmark$ & & \\
\hline Mediación educativa & 13.14 & & $\checkmark$ & & \\
\hline $\begin{array}{l}\text { Obtención de } \\
\text { información }\end{array}$ & 15 & & $\checkmark$ & & \\
\hline Masturbación infantil & 16.17 & $\checkmark$ & & & \\
\hline Claridad de información & 18.19 & $\checkmark$ & & & \\
\hline Miedos sobre sexualidad & 20 & & $\checkmark$ & & \\
\hline
\end{tabular}




\begin{tabular}{|c|c|c|c|c|c|}
\hline $\begin{array}{l}\text { Percepción de capacidad } \\
\text { de mediación. }\end{array}$ & 24 & $\bar{\checkmark}$ & & & \\
\hline $\begin{array}{l}\text { La educación sexual } \\
\text { estimula actividad } \\
\text { sexual. }\end{array}$ & 25.26 & $\checkmark$ & & & \\
\hline $\begin{array}{l}\text { La sexualidad como } \\
\text { placer }\end{array}$ & 27 & & & $\checkmark$ & \\
\hline $\begin{array}{l}\text { La heteronormatividad } \\
\text { como patrón cultural }\end{array}$ & 28 & $\checkmark$ & & & \\
\hline $\begin{array}{l}\text { Prácticas sexuales } \\
\text { exclusivas dentro del } \\
\text { matrimonio }\end{array}$ & 29 & & $\checkmark$ & & \\
\hline $\begin{array}{l}\text { Iniciativa del hombre en } \\
\text { las relaciones sexuales }\end{array}$ & 30 & $\checkmark$ & & & \\
\hline $\begin{array}{l}\text { Vida sexual activa como } \\
\text { derecho masculino }\end{array}$ & 31 & $\checkmark$ & & & \\
\hline $\begin{array}{l}\text { Vida sexual activa como } \\
\text { derecho femenino }\end{array}$ & 32 & $\checkmark$ & & & \\
\hline $\begin{array}{l}\text { Relación entre } \\
\text { enseñanza religiosa y } \\
\text { sexualidad. }\end{array}$ & 33 & & & & $\checkmark$ \\
\hline $\begin{array}{l}\text { Búsqueda de } \\
\text { información. }\end{array}$ & 34 & & $\checkmark$ & & \\
\hline $\begin{array}{l}\text { Valoración de educación } \\
\text { sexual recibida. }\end{array}$ & 35 & & $\checkmark$ & & \\
\hline $\begin{array}{l}\text { La masturbación como } \\
\text { situación perniciosa }\end{array}$ & 36 & $\checkmark$ & & & \\
\hline $\begin{array}{l}20 \text { aspectos investigados } \\
(100 \%)\end{array}$ & $\begin{array}{c}24 \\
\text { ítems }\end{array}$ & $\begin{array}{c}11 \\
55 \%\end{array}$ & $\begin{array}{c}7 \\
35 \%\end{array}$ & $\begin{array}{c}1 \\
5 \%\end{array}$ & $\begin{array}{c}1 \\
5 \%\end{array}$ \\
\hline
\end{tabular}

Cuadro 4: Niveles de significatividad del impacto del PSIF del grupo experimento de padres y madres de familia en relación con el grupo control y en relación de su propio desarrollo

\begin{tabular}{cccccc}
\hline $\begin{array}{c}\text { Aspectos de } \\
\text { investigación }\end{array}$ & $\begin{array}{c}\text { Número } \\
\text { de Ítems }\end{array}$ & \multicolumn{5}{c}{$\begin{array}{c}\text { C. } \\
\text { D. Grupo experimento } v \text { G } \text { Grupo control }\end{array}$} \\
\cline { 3 - 6 } & & $\begin{array}{c}\text { Muy } \\
\text { significativo }\end{array}$ & $\begin{array}{c}\text { Medianamente } \\
\text { significativo }\end{array}$ & $\begin{array}{c}\text { Poco } \\
\text { significativo }\end{array}$ & $\begin{array}{c}\text { Nada } \\
\text { significativo }\end{array}$ \\
$10(100 \%)$ & 16 & 6 & 2 & 1 & 1 \\
& & $60 \%$ & $20 \%$ & $10 \%$ & $10 \%$ \\
\hline
\end{tabular}

Cuadro 5: Niveles de significatividad del impacto del PSIF del grupo experimental de estudiantes en relación con el grupo control y en relación de su propio desarrollo

En el Cuadro 4 se obtienen los resultados en términos del impacto que tuvo el PSIF en el grupo experimental, tanto del grupo control y consigo mismo. La significatividad para este

Licencia Creative Commons Atribución 4.0 Internacional (CC BY 4.0)

Revista Cátedra, 3(2), pp. 52-72, mayo-agosto 2020. e-ISSN: 2631-2875

https://doi.org/10.29166/10.29166/catedra.v3i2.2184 
estudio se estableció en cuatro rangos: Muy significativo, Medianamente significativo, Poco significativo y Nada significativo.

Para el grupo experimental de madres/padres de familia, se puede inferir que el PSIF tuvo un importante impacto con un $80 \%$ de media y alta significación. Los aspectos de escasa o ninguna significación son aquellos que no se abordaron por cuestiones de estructura curricular del programa; tal es el caso de la ausencia de la información sobre los aparatos genitales de hombre y mujer. También depende de la intencionalidad del programa como es el de la laicidad que se mantuvo en su aplicación.

A modo de resumen estadístico, para el caso del estudiantado se puede observar el Cuadro 5 donde se establece que el nivel de significación es del 60\% «Muy Significativo», 20\% «Medianamente Significativo», 10\% «Poco significativo» y 10\% «Nada significativo».

Se utilizó además el coeficiente de correlación de Spearman para relacionar los datos. Esto por cuanto el trabajo investigativo corresponde a una correlación no paramétrica: a) tiene una muestra menor a los 30 individuos, b) no tiene una distribución necesariamente normal, c) tener variables psicosociales que se cuantificaron (Camacho, 2008). La aplicación se realizó con catorce ítems cuyos indicadores contienen respuestas correctas y mejores contestaciones.



Figura 1. Correlación Spearman 1: Grupo Experimental test - Grupo Control test

Para el coeficiente Spearman 1 (Figura 1), se correlacionó al Grupo Experimental y al Grupo Control con los datos obtenidos en el test o encuesta de entrada. El coeficiente de correlación de Spearman obtenido es de 0.84 , que está entre una «correlación positiva considerable» y una «correlación positiva muy fuerte» (Cabrera, 2011, pág. 81). Los dos grupos responden de una manera muy parecida y sus características en cuanto a conocimientos, creencias y actitudes sobre la sexualidad son bastante similares.

Para el coeficiente Spearman 2 (Figura 2), se correlacionó al Grupo Experimental y al Grupo Control con los datos obtenidos en el retest o encuesta de salida. El coeficiente de correlación de Spearman obtenido es de 0,16 que se caracteriza como una «correlación positiva muy débil». Se presentan dos grupos distintos en sus saberes sobre la sexualidad,

Licencia Creative Commons Atribución 4.0 Internacional (CC BY 4.0)

Revista Cátedra, 3(2), pp. 52-72, mayo-agosto 2020. e-ISSN: 2631-2875

https://doi.org/10.29166/10.29166/catedra.v3i2.2184 
producto de su participación en el programa de educación sexual PSIF del Grupo Experimento.

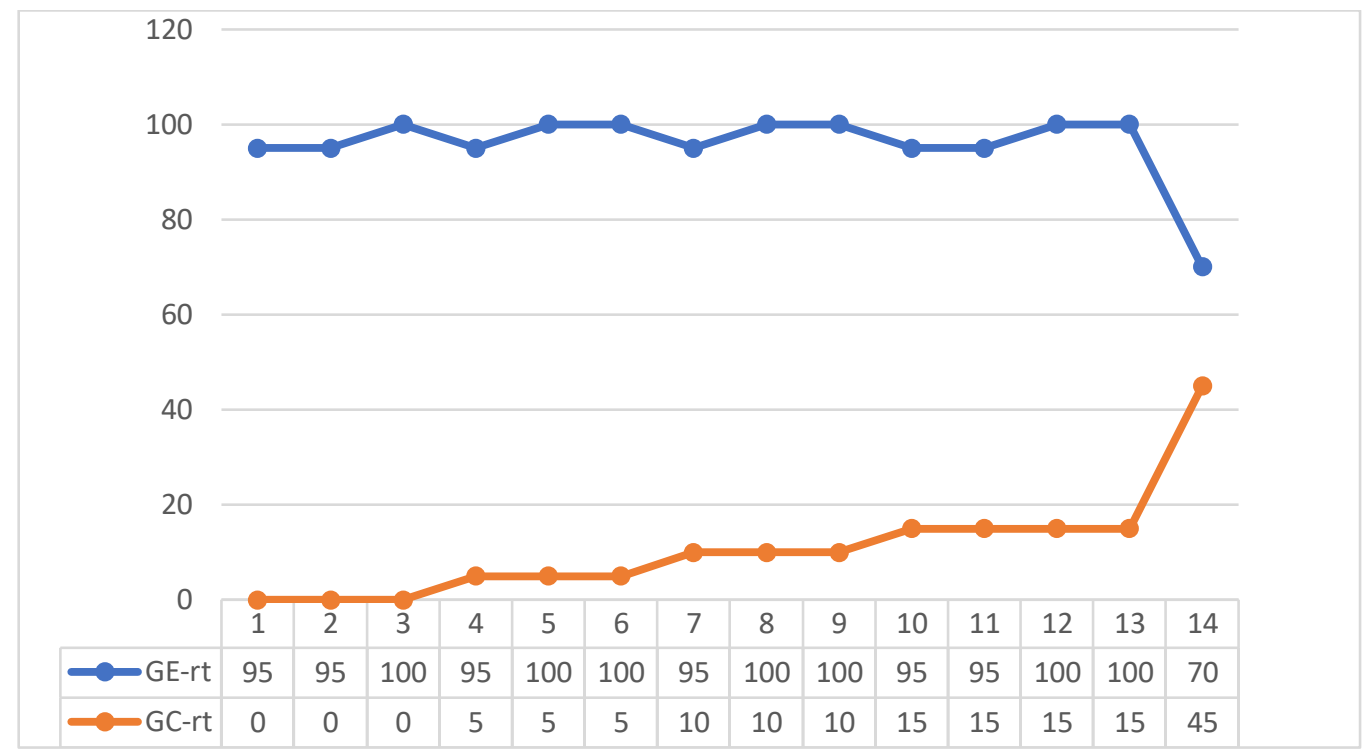

Figura 2. Correlación Spearman 2: Grupo Experimental retest - Grupo Control retest

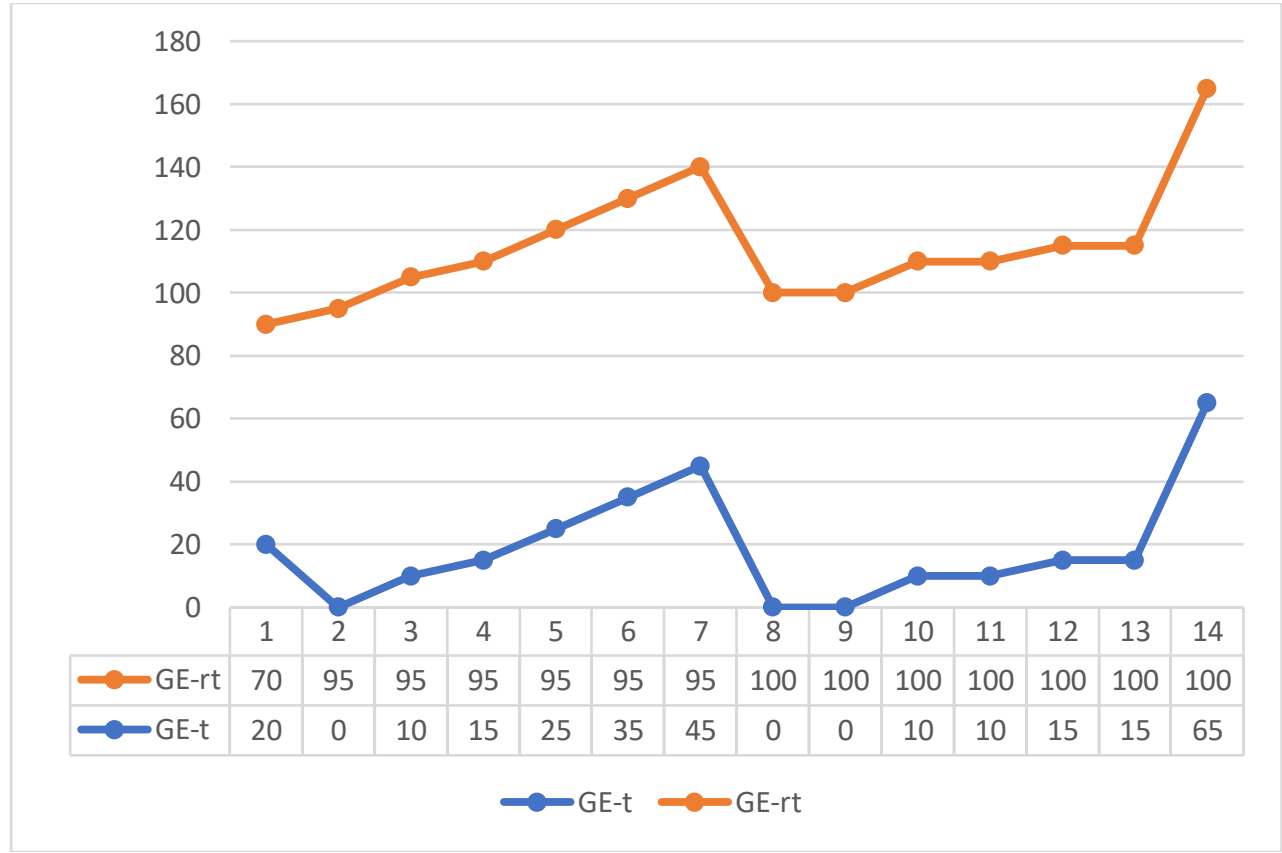

Figura 3. Correlación Spearman 3: Grupo Experimental test - Grupo Experimental retest

Para el coeficiente Spearman 3 (Figura 3), se correlacionó al Grupo Experimental en sus respuestas al testy en sus contestaciones al retest. El coeficiente de correlación de Spearman obtenido es de 0,06 que se caracteriza entre «no existe correlación alguna entre las variables» y una «correlación positiva muy débil». Las diferencias comparativas en cuanto a conocimientos, creencias y actitudes ante la sexualidad infantil y humana en general son muy amplias como impacto del PSIF. Los conocimientos, creencias y actitudes cambiaron significativamente en los dos momentos para este colectivo de madres y padres de familia. 


\section{Discusión de los resultados}

El protagonismo mayoritario de mujeres evidencia que el cuidado primario de niñas y niños infantes es obligación casi exclusiva de las madres. Por extensión se asume que esto es común en la sociedad ecuatoriana, y es producto de una división de responsabilidades con fuerte componente inequitativo de género que se mantiene en esta población local y a nivel nacional. Como precisa Royo (2011) "en las tareas domésticas como las educativas, es donde existen disparidades entre hombres y mujeres lo que constituye un reparto asimétrico del trabajo familiar" (pág. 120).

En cuanto al nivel de estudios, se infiere que cuanto mayor es el nivel de estudios, mayor es el interés por formarse como progenitores. Se debe tomar en cuenta que la adscripción al PSIF fue voluntaria. La disposición de ampliar conocimientos se explica por la necesidad de formar un acumulado educativo en cada una de estas personas.

Una de las interrogantes esenciales es conocer el criterio respecto a que, si la sexualidad de niños y niñas se desarrolla de forma natural o instintiva. Hay una preferencia inicial en madres/padres de los dos grupos de aceptar que la sexualidad se desarrolla sin la intervención de las personas adultas. Esta tendencia se repite en el GC-PMF en la encuesta de salida.

El grupo GE-PMF, pasa en el retest al 5\% en sus respuestas afirmativas lo cual se considera como muy significativo. El recorrido de aprendizaje del GE-PMF, como producto de su participación en el PSIF los lleva a considerar a la sexualidad más allá de la genitalidad, y es el resultado del abordaje del programa con la reflexión acerca que la sexualidad involucra la interrelación afectiva de las personas. Se produce en el contexto familiar y escolar, e implica la cercanía emocional del vínculo madre/padre e infante, u otros miembros familiares. Este colectivo ve a la sexualidad como el descubrimiento de sí mismos como individualidades humanas y de los demás a través de las interacciones sociales. Se asume entonces, como lo plantea Barriga-Jiménez (2013), que "la conducta sexual, como cualquier otra conducta, se moldea en el proceso de socialización desde la más tierna edad" (pág. 3). Los progenitores y progenitoras del GE-PMF toman conciencia de la importancia de esa socialización a través del PSIF.

La influencia del PSIF se hace evidente en el cambio de la percepción respecto de la edad a la que se debe iniciar el diálogo sobre sexualidad. Y según el GE-PMF debe ser desde que nace. Así, se produce una dislocación de la idea ingenua, muy generalizada, de la asexualidad de niñas/niños infantes. El grupo considera necesario que se dialogue sobre la sexualidad desde su nacimiento en una perspectiva de actuar proactivamente en lo educativo y en la prevención de conductas. Este cambio implica una ruptura a una serie de errores comunes que tienen padres/madres en sus posturas personales como fruto de una actitud social ante la sexualidad. Entre los errores están el manifiesto desinterés de las familias sobre el tema de la sexualidad, de que los infantes aprenderán solos, el argumento de que ahora tienen más oportunidades de la que los progenitores tuvieron en su época de niñez, que la sexualidad comienza en la pubertad, que esos temas los tratarán en la escuela y que las maestras les ahorrarán trabajo y preocupaciones. Font (2002), menciona además que se piensa "que la educación sexual incita a la práctica sexual, y que a sexos diferentes hay que asignar tareas diferentes" (pág. 48). Las equivocaciones devienen de los desatinos ocurridos en las propias historias individuales y familiares. Lo penoso es que en la mayoría de los casos los anacronismos han sido reforzados por el sistema educativo formal.

Otro asunto que tuvo un cambio muy significativo fue respecto a la masturbación infantil. En los datos de partida de los dos grupos y salida del GC-PMF las respuestas se dirigen hacia

Licencia Creative Commons Atribución 4.0 Internacional (CC BY 4.0)

Revista Cátedra, 3(2), pp. 52-72, mayo-agosto 2020. e-ISSN: 2631-2875

https://doi.org/10.29166/10.29166/catedra.v3i2.2184 
las conductas represivas o de una supuesta indiferencia paternal. El GE-PMF responde en el retest con alternativas más adecuadas para una intervención educativa parental que atienda el lado emocional y mejor desarrollo psicológico de niñas/niños. Ello es expresión de asumir de una manera más sensible la intervención de las personas adultas. Las reflexiones del PSIF consideraron el factor de curiosidad infantil y el autoreconocimiento corporal cuando el niño o niña se está tocando o frotando los genitales con sus manos. Además, a que haya una pausa del padre/madre para asumir una respuesta que no sea impulsiva o incluso agresiva. En el cierre del suceso, es necesario contar con un tiempo de conversación para hablar sobre la indispensable privacidad de esas conductas de tocamiento.

Queda muy claro que es posible la transformación de las creencias, conocimientos y actitudes en materia de la masturbación infantil. Esta transformación procura una educación sexual integral y armónica de niños, niñas y la familia en general. No hay diferencia en cuanto al género y las respuestas de padres/madres de familia son muy parecidas independientemente del sexo de sus hijos e hijas.

Otra transformación importante se relaciona con el diálogo intrafamiliar de los complejos temas de la sexualidad humana. El GE-PMF deriva a que este intercambio comunicacional sea claro, veraz y conciso, lo que evidencia una innovación en su estructura cognoscitiva y actitudinal. Este cambio concuerda con Luisi (2013) quien precisa que: "es necesaria una explicación más que una prohibición, de lo contrario, los niños y niñas crecen desorientados, con dudas que resolverán con la persona menos indicada y con una información no del todo correcta." (pág. 5). Estas personas la mayor parte de las ocasiones no son los padres, sino una amiga o amigo que solo alimenta la confusión.

Para atender al objetivo de indagar el empleo del lenguaje que aplican madres/padres de familia sobre la sexualidad infantil, se solicitó que escriban la designación a los órganos genitales humanos en su entorno comunitario y familiar. Las respuestas para «los órganos genitales de la mujer» fueron: chepa, chepita, chucha, conchita, cosita, cuca, cucaracha, pajarita, paloma, palomita, polla, pollita, pulpa, sapito, sapo, tórtola, vagina. Para «los órganos genitales del hombre» las contestaciones fueron: colega, cosita, culebra, culo, gorrión, güevo, huevito, huevo, mandingo, pajarito, pájaro, palito, palo, paloma, palomita, pene, penecito, penje, pepe, pepito, pincho, pipí, pito, plátano, pollito, pollo, verga, vevo,

Las denominaciones dadas son parte de la terminología inventiva e ilustrativa del acervo popular, y expresan de forma generalizada los tabúes y mitos que subsisten en la sociedad. Amaya, (2005) afirma que "de este modo se mantiene la ignorancia, la limitación del conocimiento y que la expresión humana tan esencial como la sexualidad, se desarrolle con distorsiones y desviaciones" (pág. 1). Las designaciones distintas a las que existen en el idioma español producirán confusión en niños y niñas. Por su formación, es difícil para las personas adultas pronunciar los nombres correctos de los genitales, y "seguramente se está subestimando la capacidad de aprendizaje de sus hijos e hijas infantes" (Mateo-Morales y Represas, 2007, pág. 251), lo cual se ha evidenciado en el convivir de aula por las docentes parvularias.

Existe la idea de que se protege a los niños de la sexualidad escondiendo o restringiendo la información, pero también distorsiona, como en el caso de la denominación de los genitales y que es una característica de la sociedad. "La sexualidad infantil se ha estado viendo como un problema en aumento... En la actualidad los niños que muestran un gran interés sexual continúan siendo juzgados como desviados o anormales" (Molina et al., s.f., pág. 2). La transformación de las creencias sobre la sexualidad es factible con reflexiones puntuales y profundas como las desarrolladas en el PSIF. Este proceso educativo complejo y

Licencia Creative Commons Atribución 4.0 Internacional (CC BY 4.0)

Revista Cátedra, 3(2), pp. 52-72, mayo-agosto 2020. e-ISSN: 2631-2875

https://doi.org/10.29166/10.29166/catedra.v3i2.2184 
enriquecedor permite enfrentar la problemática de información/formación sobre la sexualidad desde una perspectiva humana.

Un tema que tiende a ser aceptado incluso a nivel de educadores es que al hablar de sexo se estimula la actividad sexual de los niños, niñas y jóvenes. Esta idea es cambiada muy significativamente con el PSIF. El grupo experimental de padres y madres expresa su desacuerdo con esta afirmación que se ha constituido en un mito de la educación sexual.

Entre los temas consultados, el GC-PMF aumentó de modo considerable su temor a que su hijo o hija sea homosexual. Este incremento del miedo se explicaría por la razón del entorno ideológico que se produjo en la aprobación del matrimonio igualitario en el tiempo de la investigación. El GE-PMF se mantuvo estable en los porcentajes de respuesta. Se infiere que el grupo experimental pudo enfrentar la avalancha mediática conservadora a través de razonamientos y explicaciones personales en el desarrollo del programa educacional. Este grupo seguramente evaluó las posiciones expuestas en la resolución de la Corte Constitucional. De ese modo, asumió la que consideraron es apegada al enfoque de derechos que fue uno de los ejes del PSIF.

Dos aspectos que se desarrollaron tienen que ver con la visión androcéntrica y que se puede traducir en estrategias de enseñanza de lo sexual. Se refiere a la iniciativa sexual y a la vida sexual activa como patrimonio cultural o derecho exclusivo de los varones. Estos asuntos referencian al predominio masculino sobre la sexualidad que es naturalizado en la sociedad y aceptado por hombres y mujeres. Bourdieu (2000) precisa que "la fuerza del orden masculino se descubre en el hecho de que prescinde de cualquier justificación: la visión androcéntrica se impone como neutra y no siente la necesidad de enunciarse en unos discursos capaces de legitimarla" (pág. 24). Se ratifica el principio de heteronormatividad y el patrón cultural masculinizado que afecta a la sociedad.

Esta hegemonía arbitraria ha sido fracturada, probablemente de manera inicial pero muy evidente por el PSIF como lo demuestra la estadística comparada entre el GE-PMF y el GCPMF, y se alientan las posibilidades que con un proceso educativo se pueda trastocar esos cánones que se hallan muy profundizados. Es posible que este grupo de mujeres y hombres recorra un camino hacia la equidad de género, enfrentando normas impuestas por la sociedad patriarcal.

En los resultados estadísticos de la investigación no se evidencian diferencias de género. En primer lugar, habría que considerar hipotéticamente que existen diferencias de género en la educación sexual de infantes mujeres e infantes varones. Mateo-Morales y Represas (2007) señalan que "cuando se habla de sexualidad también se transmiten sentimientos, ideas, valores y prejuicios generados a partir de la experiencia propia" (pág. 17). Se puede razonablemente entender que las diferenciaciones de género se deslizan muy subjetivamente en el tono de voz, el tipo de palabras usadas, con las reacciones de la cara y el cuerpo, los silencios y respuestas, entre una amplia gama de posibilidades comunicativas lingüísticas y paralingüísticas. En segundo lugar, sería un interesante tema para investigar las construcciones de género en edades tempranas.

El aspecto en que el PSIF no pudo influir y de allí su nula significatividad tiene que ver con la relación entre religión y educación sexual. El programa PSIF en el que participó el GEPMF no contribuyó en este tópico, pues el proceso fue abordado desde una perspectiva laica y de respeto a las creencias religiosas de quienes asistieron. El rechazo del grupo de participantes a que las instituciones religiosas aborden la educación sexual se debe a la secularización evidenciada en la estadística. La ruptura deviene desde la misma sociedad

Licencia Creative Commons Atribución 4.0 Internacional (CC BY 4.0)

Revista Cátedra, 3(2), pp. 52-72, mayo-agosto 2020. e-ISSN: 2631-2875

https://doi.org/10.29166/10.29166/catedra.v3i2.2184 
que no ven con buenos ojos a las prácticas religiosas de sus sacerdotes, pastores, ancianos o de la misma población creyente.

A continuación, se procede a analizar y discutir la información estadística obtenida de las encuestas a estudiantes. Estas fueron realizadas en entrevistas individuales en el mismo Centro de Desarrollo Integral para la Primera Infancia Virginia Larenas. El grupo experimental de estudiantes (GE-E), son aquellos hijos e hijas que se asumen como participantes indirectos para la investigación, pues sus madres o padres asistieron a los talleres del PSIF. El grupo control de estudiantes (GC-E) fueron aquellos cuyos representantes no participaron del programa.

Para la totalidad de estudiantes en general no constituye ninguna dificultad localizar partes del cuerpo como nariz, pierna, barriga. Sin embargo, señalar sus genitales («Pene/Vulva») ya no lo consiguen con la misma facilidad en las dos encuestas de entrada y en la de salida del GC-E. Se evidencia estadísticamente que en el GE-E se desarrolló lo aprendido en el PSIF por parte de sus progenitores. Esto en el sentido de que se debe denominar a los genitales con los nombres adecuados. Además, que se puede o debe hacer de una manera espontánea, natural como se hace con las otras partes del cuerpo.

La localización y la denominación de los órganos genitales pasa de ser una prohibición tradicional a un indicador de sexualidad sana. Es posible, mediante procesos educativos de deconstrucción cultural, desaprender este tipo de proscripciones impuestas por la sociedad hacia la familia e individuos.

Cuando se averigua sobre la vergüenza al hablar de los órganos sexuales externos de hombre y mujer hay cambios importantes. El impacto significativo en el grupo experimento de padres/madres se trasladó al de sus hijos/hijas en la superación de esta actitud ante su cuerpo y fundamentalmente de sus propios órganos genitales. Hay que precisar que el GCE mantiene intacto ese temor o malestar cuando hablan del pene o la vulva en público, debido al patrón cultural imperante en su familia y entorno comunitario que da al hablar de los órganos sexuales como algo lascivo y que debe quedar oculto.

En la pregunta sobre si les gusta tocarse los genitales, la respuesta mayoritaria y total es afirmativa para los dos grupos de estudiantes. En este tema, tampoco se evidencian diferencias entre niñas y niños, no pudiendo establecerse contrastes de género en las respuestas. Los niños y niñas complementaban sus contestaciones refiriendo a que les gusta tocarse "pero que no me dejan" por parte de sus progenitores y que se verá en el siguiente aspecto.

El análisis de la información correspondiente a las respuestas de niños y niñas en referencia a lo que sus progenitores hacen al momento de la masturbación infantil, requiere una mirada cualitativa más que estadística. Buena parte de las respuestas van dirigidas al enojo parental ("Se enoja", "Se pone brava"), a cortar la conducta intempestivamente ("Que me quede quieto"), a respuestas verbales ("Me habla"). Estas reacciones parentales, quizá impulsivas son propias de la incapacidad de atender situaciones de erotismo infantil e impregnadas de actitudes y creencias adultocéntricas. Hay situaciones de inculcación del miedo ("Que me va a mandar al doctor", "Que ya le lava"). Posiblemente también hay preocupación por la salud en las respuestas del tipo: "Que si me estará picando".

En el GE-E la respuesta: "Ya no me quema las manos...ya nos perdonamos" de un estudiante, sorprendió por la emotividad manifiesta. La situación, fue corroborada en medio del llanto de la madre. Ella, antes de su participación en el PSIF, cuando veía a su hijo tocando sus genitales, tomaba una cuchara, la calentaba en una hornilla de la cocina y quemaba las manos del niño. La madre entendió, luego del programa, que la masturbación, como Licencia Creative Commons Atribución 4.0 Internacional (CC BY 4.0) 
exploración y satisfacción de la curiosidad infantil de su propio cuerpo, de ninguna manera era perniciosa para su hijo. Por ello la madre pidió perdón al hijo, los dos se abrazaron y lloraron ante la experiencia anterior y posterior.

La mayoría de las respuestas que emiten los niños y niñas del GE-E indican, que sus madres y padres tienen una actitud más inclusiva y humana frente a la masturbación infantil: "Me dice que sí me toque", "Que sólo yo me puedo tocar el pene", "Ya no me pega", "Dice que debo tocarme solito", "Ya no me habla". La actitud maternal y paternal que se hallan detrás de las respuestas infantiles también evidencian que hay un puente comunicacional, y brinda la posibilidad de tener niñas, niños y jóvenes que puedan hablar con sus progenitores de sexualidad con mayor apertura y confianza.

A la pregunta "¿Cómo has escuchado que le dicen a "hacer el amor"?», aproximadamente la mitad del estudiantado de los dos grupos de infantes, en el test y retest, responden: "Nada" "No sabe/No responde papá/mamá". El menosprecio a las relaciones sexuales ya se expresa en esta edad cuando responden "Cochinadas" o "Púchilas". Expresar al acto sexual en frases coloquiales como "Cositas" o "Cuchicuchi", supone de todos modos esconder y mantener en la invisibilidad a estas relaciones humanas. El hecho de que el 20\% en el GE-E respondan expresiones del tipo "Quererse las personas" y "Hacer el amor", puede indicar una estadística algo significativa en el impacto del PSIF y que se produjo un cambio cognitivo al respecto.

Sobre el nacimiento de las personas, el GE-E en el test y el GC-E en las dos encuestas dan respuestas evasivas o de desconocimiento directo. Las opciones mayoritarias son: "No se dice”, "No dice nada”, "Me sacaron de la barriga” y "No sabe/No responde papá/mamá". En el retest, el GE-E la totalidad de las contestaciones: "Por la vagina de mamá", "Le cortaron la barriga a mi mami" y "Por la barriga», dan cuenta de un lenguaje más preciso. Se asume que es producto de una comunicación más adecuada por parte de los progenitores que participaron en el PSIF.

Sobre el proceso de fecundación, el GE-E en el test no da ninguna respuesta que pueda ser considerado adecuada o correcta. En el retest, la mayoría de este grupo de estudiantes afina considerablemente las respuestas: "El papá le pone el bebé, le pone dentro el espermatozoide en la barriga", "Cuando hacen el amor, el papá le pone la semilla", "Por la semilla que le pone mi papi", "Por la vagina se meten, el papá le pone la semillita". En el GCE de modo general, las respuestas en las dos encuestas son inexactas e inapropiadas. Se evidencia un manejo conceptual más exacto en el GE-E luego de que sus padres y madres participaran del PSIF. Uno de los ejes que este programa enfatiza justamente es la calidad de mensajes que se expresan en medio de un ambiente de calidez y confianza indispensables.

\section{Conclusiones}

El objetivo general fue verificar si mediante un proceso formativo cambian las formas como padres/madres de familia informan sobre la sexualidad a sus hijos e hijas. La conclusión final es que, mediante un programa educativo adecuado, se cambian los conocimientos, creencias y actitudes de madres y padres de familia, pues se brindan herramientas y estrategias para construir una sexualidad enriquecedora en niños y niñas infantes. Estos elementos se concretizan en diferentes aspectos que constituyen los objetivos específicos.

En relación al objetivo específico: «Indagar el empleo del lenguaje que aplican las madres y padres de familia sobre la sexualidad infantil», se concluye que la mayoría de las madres y padres de familia encuestados utilizan términos y frases distintos a los establecidos en el

Licencia Creative Commons Atribución 4.0 Internacional (CC BY 4.0)

Revista Cátedra, 3(2), pp. 52-72, mayo-agosto 2020. e-ISSN: 2631-2875

https://doi.org/10.29166/10.29166/catedra.v3i2.2184 
idioma español. El uso de estas expresiones en la cotidianidad obedece a la inseguridad que los progenitores y progenitoras tienen respecto de sus conocimientos sobre la sexualidad, pues este tópico sigue considerándose un tema tabú en la sociedad. En ese sentido el vocabulario tiende a ocultarse en un lenguaje popular, folclórico, escindido de la ciencia.

En lo referente al objetivo específico «Conocer las estrategias materno-parentales que utilizan madres y padres de familia para la educación sexual de niñas y niños infantes», se obtienen dos grupos de conclusiones: 1) las que aplican madres y padres de familia para la educación sexual de niñas y niños infantes del grupo experimental antes del PSIF y del grupo control antes y después de las encuestas, y, 2) las que aplican el grupo experimental después del PSIF.

Dentro de las estrategias del primer grupo, se concluye:

Sobre diversos temas de la sexualidad, el estudiantado respondió de un modo incorrecto o inadecuado, debido a las evasivas o respuestas indirectas que sus progenitores y progenitoras dan a sus inquietudes precisas. La prohibición de tratar el tema de la sexualidad o situaciones conexas es forma común de esconder le información, lo que alienta a una deformación sobre la sexualidad de la niñez, por parte de la absoluta mayoría de padres/madres de familia.

Cuando la evasión y las prohibiciones son insuficientes, la represión física o intelectual es la estrategia directa de imponer el silencio o el miedo alrededor de los tópicos de la sexualidad por parte de la madre/padre de familia en su casa. Las comparaciones o similitudes de los órganos sexuales humanos con objetos o animales del entorno son una manera pseudoeducativa de inculcación sobre la sexualidad.

Dentro de las estrategias del segundo grupo, de quienes participaron en el PSIF, hay varias transformaciones. Se concluye que las madres y padres de familia aplicaron, durante y después del proceso, el diálogo como una forma puntual y directa de abordar las inquietudes infantiles sobre sexualidad. Implementaron momentos de conversación por propia iniciativa materno-parental y no solamente de manera reactiva ante la curiosidad infantil. Hay reflexión en términos de una comunicación bidireccional enriquecedora, de mutuo aprendizaje y saludable. Esta estrategia es su nueva forma de asumir la información y la formación sexual con sus vástagos durante el proceso de participación en el PSIF.

Se evidencia un manejo conceptual más preciso en los estudiantes hijos e hijas de los padres y madres que participaron del PSIF. La claridad y la concreción de las respuestas son dos de las características esenciales de la interrelación comunicativa que el grupo experimento asume como fundamental en el proceso de enseñanza y aprendizaje sobre sexualidad en el ámbito familiar.

Uno de los objetivos investigativos es «Describir los conocimientos, creencias y actitudes sobre la sexualidad que tienen los niños y niñas infantes». Quienes participaron del programa afirman que es necesaria la intervención de las personas adultas en la educación y desarrollo integral de la sexualidad infantil, pues el proceso formativo determina que se considere a la sexualidad como una construcción social y que esos aprendizajes pueden ser motivadores para la niñez.

La autopercepción de sus propias capacidades comunicativas cambia radicalmente en el grupo de madres/padres de familia, luego de su participación en el PSIF. El grupo control se mantiene en sus ideas originales, mientras que el grupo experimental busca las respuestas adecuadas si es que no las conoce, y está predispuesto a comprender posiciones ideológicas

Licencia Creative Commons Atribución 4.0 Internacional (CC BY 4.0)

Revista Cátedra, 3(2), pp. 52-72, mayo-agosto 2020. e-ISSN: 2631-2875

https://doi.org/10.29166/10.29166/catedra.v3i2.2184 
que existen en esta materia e intercambiar informaciones y saberes incluso dentro de su entorno familiar o comunitario.

El PSIF como programa de reaprendizaje, permite el cambio de actitud ante la heterosexualidad como norma social. El grupo experimental demuestra una actitud tolerante e inclusiva que rompe lo impuesto por la estructura ideológica-educativa. En esta línea, concuerda que la masturbación o tocamiento físico de los genitales por parte de niñas y niños es parte de una construcción saludable de la sexualidad infantil. Tres tópicos se colocan en prospectiva para futuras investigaciones desde este trabajo. El primero, caracterizar la forma de construcción del género en edades iniciales del ser humano. El segundo, determinar la influencia de la religión en aspectos como la sexualidad u otros que tienen que ver con la educación de niñas, niños y jóvenes. El tercero, definir la construcción de la sexualidad infantil con poblaciones más amplias y diferentes social, económica y culturalmente. Estos asuntos deben analizarse con equipos multidisciplinarios que aborden desde distintas perspectivas el fenómeno de la sexualidad humana temprana. 


\section{Bibliografía}

Alcántara, E. (2012). Identidad sexual / rol de género. Recuperado el 18 de agosto de 2019 de: http://www.debatefeminista.cieg.unam.mx/wpcontent/uploads/2016/03/articulos/047 10.pdf

Amaya, R. (2005). Mitos y tabúes sobre la sexualidad. Curso de Formación de Orientadoras y Educadoras en Sexualidad Humana. Recuperado el 11 de julio de: http://www.educacionsexual.com.ar/biblioteca-online/mitos-y-tabues-sobre-lasexualidad

Barriga-Jiménez, S. (2013). La sexualidad como producto cultural. Perspectiva histórica y psicosocial. Anduli - Revista Andaluza de Ciencias Sociales № 12 - 2013. Recuperado el 26 de septiembre de 2019 de:

https://dialnet.unirioja.es/descarga/articulo/4772257.pdf

Bourdieu, P. (2000). La dominación masculina. Editorial Anagrama. Barcelona. Recuperado el 20 de julio de 2019 de: https://www.ocac.cl/wpcontent/uploads/2015/01/Pierre-Bourdeu-La-dominaci\%C3\%B3n-masculina.pdf

Cabrera, M. (2011). Inteligencia emocional y rendimiento académico de los alumnos del nivel secundario de una institución educativa de la región Callao. Tesis. Universidad San Ignacio de Loyola. Recuperado el 12 de marzo de 2019 de: http://repositorio.usil.edu.pe/handle/123456789/1120

Camacho, J. (2008). Asociación entre variables: correlación no paramétrica. Nota estadística. Recuperado de: https://www.scielo.sa.cr/pdf/amc/v50n3/3783.pdf

Consejo Nacional para la Igualdad de Género. (2018). Guía de orientaciones técnicas para prevenir y combatir la discriminación por diversidad sexual e identidad de género en el sistema educativo nacional. Publicación con apoyo de Agencia Flamenca de Cooperación Internacional - VVOB. Quito.

Font, P. (2002). Pedagogía de la Sexualidad. Instituto de Ciencias de la Educación de la Universidad de Barcelona y Editorial Grao. Barcelona, España.

Hernández, G. y Jaramillo, C. (2003). La educación sexual de la primera infancia. Guía para madres, padres y profesorado de Educación Infantil. Recuperado el 6 de julio de 2019 de:

https://ioc.xtec.cat/materials/FP/Materials/1752 EDI/EDI $1752 \mathrm{M} 07 /$ web/html/ WebContent/u2/media/educacio de la sexualitat en la infancia mec.pdf

Lamas, M. (2000). Diferencias de sexo, género y diferencia sexual. Cuicuilco, vol. 7, núm. 18, enero-abril, 2000. Escuela Nacional de Antropología e Historia.

Luisi, V. (2013). Educación de la sexualidad en el contexto familiar y escolar. Educere, vol. 17, núm. 58, septiembre-diciembre, 2013, pp. 429-435. Universidad de los Andes. Mérida, Venezuela. Recuperado el 2 de marzo de 2019 de: https://www.redalyc.org/pdf/356/35630404006.pdf 
Mateo-Morales, M. y Represas, B. (2007). ¿Cómo se lo explico? La educación sexual en la infancia. Editorial Síntesis. Madrid.

Ministerio de Educación y Cultura. (2006). Plan nacional para la erradicación de los delitos sexuales en el ámbito educativo. Recuperado el 12 de febrero de 2019 de:

https://evaw-global-

database.unwomen.org/en/countries/americas/ecuador/2006/plan-nacional-deerradicacion-de-los-delitos

Ministerio de Educación. (2015). Programa Educando en Familia. Educación de la sexualidad y afectividad. Guía para formadores. Quito. Recuperado el 6 de enero de 2020 de: https://educacion.gob.ec/wp-content/uploads/downloads/2017/05/1.Guia-Sexualidad Formadores.pdf

MINEDUC-VVOB. (2019). Guía para fomentar la inclusión de hombres y mujeres en la gestión escolar y en el desarrollo profesional directivo y docente de Bachillerato Técnico. Primera edición. Ministerio de Educación del Ecuador y Agencia Flamenca para el Desarrollo-VVOB. Quito.

Molina, M., Baeza, E., Corbella, A. y Bassas, N. (s.f.). Aproximación teórica a la sexualidad infantil. Recuperado el 10 de marzo de 2019 de:

http://www.centrelondres94.com/files/APROXIMACION TEORICA A LA SEXUALID AD INFANTIL 0. pdf

Morales, P. (2013). Androcentrismo y discurso radiofónico: lo juvenil en la matriz de la inteligibilidad mediática. Recuperado el 4 de agosto de 2019 de http://sedici.unlp.edu.ar/bitstream/handle/10915/41594/Documento completo.p $\underline{\mathrm{df} \text { ? }}$ equence $=1$ \&isAllowed $=\mathrm{y}$

Moreno, J. (2019). La carta robada. Un estudio sobre la (no)percepción de la heteronormatividad en el ámbito educativo. Discurso \& Sociedad, Vol.13(4), 2019, 556-574. Recuperado el 5 de julio de 2019 de http://www.dissoc.org/ediciones/v13n04/DS13(4)MorenoSanchez.pdf

Murillo, J. (s.f.). Métodos de investigación de enfoque experimental. Recuperado el 2 de mayo de 2019 de: http://www.postgradoune.edu.pe/pdf/documentosacademicos/ciencias-de-la-educacion/10.pdf

Organización Mundial de la Salud. (2018). La salud sexual y su relación con la salud reproductiva: un enfoque operativo. Recuperado el 4 de junio de 2019 de: https://apps.who.int/iris/bitstream/handle/10665/274656/9789243512884spa.pdf

Registro Oficial. Órgano del Gobierno del Ecuador. Año II - Quito, miércoles 29 de septiembre del 2004 - № 431. Recuperado el 18 de febrero de 2019 de: https://www.registroficial.gob.ec/index.php/registro-oficialweb/publicaciones/registro-oficial/item/6151-registro-oficial-no-431 
Registro Oficial Órgano del Gobierno del Ecuador. Año II - Quito, viernes 27 de octubre del 2006 - № 386. Recuperado el 20 de febrero de 2019 de: https://www.registroficial.gob.ec/index.php/registro-oficialweb/publicaciones/registro-oficial/item/5108-registro-oficial-no-174

Registro Oficial. Órgano del Gobierno del Ecuador. Año I - Quito, lunes 17 de septiembre de 2007 - № 171. Recuperado el 18 de febrero de 2019 de: Año I - Quito, lunes 17 de septiembre de 2007 - № 171

Registro Oficial - Edición Especial № 529-miércoles 29 de agosto de 2018 - 3. Acuerdo Interministerial no. 0247 - 2018. Recuperado el 15 de marzo de 2019 de: https://www.derechoecuador.com/uploads/content/pdf/2018/12/pdf181203053 944 1543876796.pdf

Royo, R. (2011). Maternidad, paternidad y conciliación en la CAE. ¿Es el trabajo familiar un trabajo de mujeres? Universidad de Deusto. Bilbao, España. Recuperado el 17 de junio de 2019 de: http://www.deustopublicaciones.es/deusto/pdfs/otraspub/otraspub02.pdf

Rosso, N. (2016). El sistema patriarcal: sus fundamentos y funcionamiento. Recuperado el 18 de julio de 2019 de https://www.academia.edu/30940159/El sistema patriarcal sus fundamentos y f uncionamiento

Suárez, J. (2016). Glosario de la diversidad sexual, de género y características sexuales. Consejo Nacional para Prevenir la Discriminación. México. Recuperado el 28 de agosto de 2019 de:

https://www.conapred.org.mx/documentos cedoc/Glosario TDSyG WEB.pdf

\section{Autoras}

MARTHA QUIROLA-LARREA obtuvo su título de Licenciatura en Ciencias de la Educación, mención profesora parvularia en la Universidad Central del Ecuador, el 2015. Estudia una maestría en Educación Inicial de la misma universidad.

Actualmente es docente titular en la Escuela de Educación Básica Fiscal Aurelio Chiriboga.

CECILIA JARAMILLO-JARAMILLO obtuvo su título de Licenciada en Ciencias de la Educación, especialización Filosofía, Ciencias Sociales y Económicas y la Maestría en Educación Superior en la Universidad Central del Ecuador. Curso sobre Género, Desarrollo y Derechos Humanos.

Actualmente es docente de la Universidad Central del Ecuador, Facultad de Filosofía, Letras y Ciencias de la Educación - Carrera en Pedagogía de la Historia y las Ciencias Sociales Programas de maestría. 